\title{
On the validity of ideal MHD in the vicinity of stagnation points in the heliosphere and other astrospheres
}

\author{
D. H. Nickeler and M. Karlický \\ Astronomical Institute Ondřejov AV ČR, Czech Republic
}

Received: 22 August 2007 - Revised: 13 November 2007 - Accepted: 18 January 2008 - Published: 5 March 2008

\begin{abstract}
The paradigm of ideal MHD is investigated in the vicinity of null points of flows and magnetic fields. These null points determine the location and geometrical shape of the heliopause (or other astropauses). We investigate the question whether regular and stable solutions of the ideal MHD equations in the vicinity of null points of flow and magnetic field exist. This is done to test the validity of ideal MHD in the vicinity of flow and magnetic field of the plasma boundaries of stellar winds and their local interstellar medium. We calculate the general solutions of ideal MHD in the vicinity of magnetic null points and use the standard form of stagnation point flows to analyse all possible time evolutions of these plasma environments. We show that the solution space in 2-D consists almost exclusively of either exponentially (in time) growing velocity or magnetic fields, or collapse solutions. Regular solutions must be three-dimensional and seem to be unstable with respect to small perturbations. This is an argument that reconnection has to take place in such regions and that therefore nonideal terms in Ohm's law are necessary, allowing for reconnection. We conclude that the use of ideal MHD in the vicinity of singular points of flow and magnetic field has to be analysed very carefully with respect to simulation results as those simulations show numerical dissipation (resistivity). These simulations can therefore produce unphysical reconnection regimes. Thus one has to search for a realistic Ohm's law, allowing for reconnection at the heliospheric boundaries.
\end{abstract}

\section{Introduction}

The solar wind is interacting with its surrounding interstellar medium (ISM) leading to the formation of the so-called heliopause (or astropause in more general). This heliopause is

Correspondence to: D. H. Nickeler

(nickeler@asu.cas.cz) separating the solar wind plasma from the ISM plasma, forcing the ISM material to flow around the obstacle formed by the solar wind. At a certain point, the nose of the heliopause, the velocity of the ISM plasma flow must vanish: this point is called a stagnation point (see, e.g. Parker, 1963; Nickeler et al., 2006). The heliopause is mathematically speaking a separatrix. The classical heliospheric definitions are given, e.g. in Parker (1963) and Fahr et al. (1993) or as an approximation in Fahr et al. (1988). Another approach, additionally taking the stability of the heliopause flanks into consideration, was performed by Baranov et al. (1992). In the aforementioned articles it is emphasized that the heliopause is a free pressure equilibrium surface. A more precise topological definition (on which our investigation is based) of such a separating surface or separatrix is presented, e.g. in Arnold (1992); Reitmann (1996). In general, the streamlines (or field lines) of a vector field are equivalent to the phase portrait of a corresponding dynamical system. The stagnation point is the intersection of the 1-D-stable manifold (= the stagnation line) with the 2-D-unstable manifold (= separatrix surface or pause) in general 3-D problems (see, e.g. Arnold, 1992; Reitmann, 1996). The stable manifold consists of two streamlines of opposite direction, intersecting at the location where the velocity vanishes: the stagnation point. The unstable manifold is the separatrix surface, where the streamlines leave, i.e. point out of the stagnation point and flow outwards. Therefore in heliospheric physics the heliopause is defined as the 'hydropause', separating the two different plasmas, namely the solar plasma and the interstellar plasma. The disadvantage of this definition is that in a multifluid plasma there are different 'pauses' due to the different species, respectively fluids, e.g. neutrals, ions, electrons. Therefore a better definition of the heliospheric boundary maybe the magnetopause. This point will be discussed in Sect. 3.

From the topological point of view the magnetopause has the same definition as the hydropause: both are separating

Published by Copernicus Publications on behalf of the Arbeitsgemeinschaft Extraterrestrische Forschung e.V. 
surfaces. In the case of magnetic field lines an inversion of this image is possible, i.e. here the 2-D manifold may also be the stable manifold and the 1-D manifold the unstable one, see Parnell et al. (1996). The eigenvalues of the linearized vector field determine the type of null point, see Parnell et al. (1996). Then the question arises, whether in ideal MHD stationary solutions exist, which permit that the two types of separatrix surfaces are identical, or whether they have to be different. We show that in 3-D locally such an identity must be fulfilled, but the question is whether the found solutions are stable.

But to find regular solutions of the MHD equations in the vicinity of singular points of flow (= stagnation point) and magnetic field (=null or neutral point) is a problematic task. In the case of 2-D configurations, it is obvious that the poloidal components of magnetic and flow field have to be parallel (see, e.g. Nickeler and Karlický, 2006; Contopolous, 1999). Otherwise either at least one of the vector components of the magnetic field or of the velocity field has to diverge as shown, e.g. by Nickeler and Karlický (2006).

It is often stated that null line or null sheets of the magnetic field exist, if ideal MHD codes are used to calculate a fluid model for the heliosphere, see e.g. Zank (2007). In contrast to that it has been proven by Hornig and Schindler (1996) that configurations containing lines, surfaces, or regions where $\boldsymbol{B}=\mathbf{0}$, have an unstable topology, i.e. no flux conserving flow like in ideal MHD exists.

To represent a model heliosphere it is necessary to ask, whether it is possible to calculate quasi-stationary MHD states (non-unstable/decaying, non-diverging) at the noses of astrospheres, i.e. in the vicinity of the stagnation and null points of the large scale heliospheric MHD fields. A similar task, concerning the stability of tangential discontinuities with a stagnation point in hydrodynamics, was carried out by Belov (1997), see also the review paper by Ruderman (2000). These authors also found instability in the vicinity of the stagnation point for a tangential discontinuity, assuming a potential flow.

Another application for small velocity fields can be found in pre-solar-flare stages. Thus we make assumptions, allowing only for small velocity fields compared to the speed of sound: Is it then possible to find (asymptotical) stationary flows or intermittent/oscillatory flows? In Sect. 2 we restrict our investigation to general solutions of ideal Ohm's law in the vicinity of null points of magnetic or velocity field, assuming that global regular and bounded solutions exist. We find restrictions for the (general) solution of the ideal Ohm's law using characteristic method by inserting the characteristic solutions into mass continuity equation and Euler equation. Finally, we want to shed light on the problem of stationary ideal MHD solutions in the vicinity of 3-D magnetic null points (Sect. 3).

\section{Illustrative example of the problem: the potential magnetic null point and the stagnation point of sub- sonic flows}

The distribution of null points of a vector field determines the global topology and the geometry or shape of the outermost separatrix: the pause. Singular points seem to be a natural 'ingredient' in different heliophysical scenarios: (i) in the scenario of the quasi-stationary heliosphere the global shape of field lines and stream lines is determined by the structure of field- and streamlines in the vicinity of the stagnation point; (ii) in the case of the quasi-static magnetic structures in the solar corona, the important null points are the magnetic ones. As in the vicinity of magnetic nulls or stagnation points in 2-D no stationary flows can exist that are not field aligned, see Nickeler and Karlický (2006), we search for oscillating structures.

To avoid the occurence of magnetic instabilities we take a null point without free magnetic energy: an example of magnetic structures are potential fields in the solar corona. The plasma $\beta$ is small in the solar corona, according to the averaged values of pressure $p: p \approx 2 \times 10^{-2} \mathrm{~Pa}$, and the magnetic field $B \approx 10^{-2} \mathrm{~T}$, thus $\beta=p /\left(B^{2} / 2 \mu_{0}\right) \approx 5 \times 10^{-4}$. Therefore it is often argued that the pressure gradient is zero, i.e. it can be neglected, and thus the Euler equation can be neglected (so called kinematic approach, where only the Laplace equation and the ideal Ohm's law are solved). If we regard the vicinity of magnetic neutral points the plasma $\beta$ converges to infinity, in contrast to the before shown estimations. Thus the pressure gradient cannot be neglected.

\subsection{Assumptions and basic MHD equations}

If we want to find a quasi-static solution for the ideal MHD equations in the vicinity of null points, the velocity field (e.g. oscillating structures) should be small compared to typical velocities like the speed of sound (the Alfvén velocity is zero at the null point). The nonlinear part of the Euler equation can be neglected but not the partial derivative, implying $\mathrm{v} \ll \mathrm{v}_{S}, \boldsymbol{v} \cdot \boldsymbol{\nabla} \approx 0$ and $\partial / \partial t \neq 0$. In addition we assume a barotropic law $p=p(\rho)$ and solve the problem in pure $2-D$, i.e. $\partial / \partial z=0$, but all variables are in principle (parametrically) time dependent. Based on the aforementioned assumptions we introduce the set of equations that has to be solved in the vicinity of null points: The equation of mass continuity Eq. (1), the barotropic law Eq. (2), the Euler or momentum equation Eq. (3), the $z$-component of ideal Ohm's law Eq. (4) that guarantees flux and field line freezing, and the Laplace equation Eq. (5)

$$
\begin{aligned}
\frac{\partial \rho}{\partial t}+\rho \boldsymbol{\nabla} \cdot \mathbf{v} & =0, \\
p & =p(\rho), \\
\rho \frac{\partial \mathbf{v}}{\partial t} & =-\nabla p,
\end{aligned}
$$




$$
\begin{aligned}
\frac{\partial A}{\partial t}+\mathbf{v} \cdot \nabla A & =0, \\
\Delta A & =0,
\end{aligned}
$$

where $A$ is the magnetic flux function, i.e. the $z$-component of the vector potential of the magnetic field, writing $\boldsymbol{B}=\boldsymbol{\nabla} \times\left(A \boldsymbol{e}_{z}\right)=\boldsymbol{\nabla} A \times \boldsymbol{e}_{z}=\nabla \phi_{m}, p$ is the thermal pressure, $\rho$ the mass density and $\mathbf{v}$ the plasma velocity. The Laplace equation, Eq. (5), reflects the fact that $\boldsymbol{\nabla} \times \boldsymbol{B} \equiv \mathbf{0}$, i.e. that the magnetic field is a potential field and therefore current-free, thus these fields do not contain free magnetic energy. From the Euler equation Eq. (3) and the barotropic law Eq. (2) we can conclude, that the curl of the plasma velocity vanishes $(\boldsymbol{\nabla} \times \mathbf{v}=\mathbf{0})$ and that thus the velocity can be expressed as a gradient $\mathbf{v}=\nabla \varphi$ i.e. the velocity field is curlfree and can therefore be written as a gradient of the velocity potential $\varphi$. With the help of the velocity potential $\varphi$ we can rewrite the system of Eqs. (1) - (5) as

$$
\begin{aligned}
g^{\prime}(\dot{\varphi}) \ddot{\varphi}+g(\dot{\varphi}) \Delta \varphi & =0, \\
\rho & =g(\dot{\varphi}), \\
\nabla \varphi \cdot \nabla A & =-\frac{\partial A}{\partial t}, \\
\Delta A & =0,
\end{aligned}
$$

where the density $\rho$ can be expressed as a function of the time derivative of the velocity potential $\varphi$. We point out that Eq. (7) is a consequence of Bernoulli's theorem

$\dot{\varphi}+\frac{1}{2}|\nabla \varphi|^{2}+W(\rho)=$ const,

where

$W(\rho)=\int \frac{d p}{\rho}$

is the gas enthalphy, neglecting the small specific flow kinetic term $\mathbf{v}^{2} \equiv|\nabla \varphi|^{2}$. To solve the system (6)-(9) of quasi-static MHD equations either boundary conditions or constraints must be prescribed by us. We use the constraint of a potential magnetic $X$-point in the next section.

2.2 Example: the standard form of a potential field in the vicinity of a magnetic neutral point

We take the potential approximation to avoid instabilities that occur due to free magnetic energy. Low energy fields usually do not show unstable behaviour.

With $A(x, y, t)=A_{0}(t) x y$ we find the general solution of ideal Ohm's law, Eq. (8), by applying the method of characteristics, see e.g. Bronstein and Semendjajew (1979),

$$
\begin{gathered}
\frac{\partial A}{\partial x} \frac{\partial \varphi}{\partial x}+\frac{\partial A}{\partial y} \frac{\partial \varphi}{\partial y}=-\frac{\partial A}{\partial t} \\
\Rightarrow A_{0} y \frac{\partial \varphi}{\partial x}+A_{0} x \frac{\partial \varphi}{\partial y}=-\dot{A}_{0} x y
\end{gathered}
$$

$$
\begin{aligned}
& \Rightarrow d y: d x=A_{0} x: A_{0} y \text { and } d \varphi: d x=\frac{\left(-\dot{A}_{0}\right) x y}{A_{0} y} \\
& \Rightarrow \zeta:=\varphi+G \frac{x^{2}}{2} \text { and } \xi:=x^{2}-y^{2}
\end{aligned}
$$

where $\xi=$ const, and $\zeta=$ const are the equations of the characteristics and $G:=\frac{\dot{A}_{0}}{A_{0}}$ is the specific flux growth rate. Thus for a potential field $A=A_{0}(t) x y$, the ideal Ohm's law Eq. (8) has the general solution

$\varphi=f(\xi, t)-\frac{G x^{2}}{2}$,

where $\xi:=x^{2}-y^{2}$, and $f$ is an arbitrary function of $\xi$ and $t$ and can only be determined by initial or boundary conditions or additional equations, like Eq. (6) (we will follow this way to solve the quasi-static system simultaneously). We can use the above general solution of the ideal MHD induction equation Eq. (8) to restrict the solution of the complete ideal MHD system. This can be done by inserting the general solution Eq. (16) into the mass continuity equation Eq. (6).

\subsection{Case I: Isothermal approach}

In the isothermal case $p(\rho)=\frac{k_{B}}{\mu} \rho T_{0}$ is the equation of change of state with $T_{0}$ as constant temperature. Thus we can infer that

$p^{\prime}(\rho)=\frac{k_{B}}{\mu} T_{0} \Rightarrow g(\dot{\varphi})=\rho_{0} \exp \left[-\mu \frac{\dot{\varphi}-\dot{\varphi_{0}}}{k_{B} T_{0}}\right]$.

Inserting Eq. (17) into Eq. (6) results in

$-\frac{\mu g(\dot{\varphi})}{k_{B} T_{0}} \ddot{\varphi}+g(\dot{\varphi}) \Delta \varphi=0 \Rightarrow-\frac{1}{v_{S}^{2}} \ddot{\varphi}+\Delta \varphi=0$,

with the definition $v_{S}^{2}:=\frac{k_{B} T_{0}}{\mu}$. The velocity $v_{S}$ is the isothermal sound speed. The general solution of the differential equation Eq. (18) is obtained, inserting the general solution of the ideal Ohm's law, Eq. (16), into the linear wave equation Eq. (18). This gives

$$
\begin{aligned}
4\left(x^{2}+y^{2}\right) f^{\prime \prime}-G(t)-\frac{1}{v_{s}^{2}}\left(\ddot{f}-G x^{2} / 2\right) & =0 \\
\Rightarrow 4\left(2 x^{2}-\xi\right) f^{\prime \prime}-G(t)-\frac{1}{v_{s}^{2}}\left(\ddot{f}-G x^{2} / 2\right) & =0,
\end{aligned}
$$

where the prime denotes the derivatives with respect to $\xi$ and like usual dots the time derivatives. As $\xi, x$ and the time $t$ are independent coordinates of the problem one can sort the terms and recognize that

$$
\begin{gathered}
f^{\prime \prime}=\frac{\ddot{G}}{-16 v_{s}^{2}}:=f_{0}(t) \\
\Rightarrow \quad f=\frac{1}{2} f_{0}(t) \xi^{2}+f_{1}(t) \xi+f_{2}(t)
\end{gathered}
$$


is a necessary assumption to guarantee that the term with $x^{2}$ vanishes. The remaining equation is

$$
\begin{gathered}
\quad-4 \xi f_{0}-G-\frac{1}{v_{s}^{2}} \ddot{f}=0 \\
\Rightarrow \quad-4 \xi f_{0}-G-\frac{1}{v_{s}^{2}}\left[\frac{1}{2} \ddot{f}_{0} \xi^{2}+\ddot{f}_{1} \xi+\ddot{f}_{2}\right]=0 \\
\Rightarrow G=-\frac{1}{v_{s}^{2}} \ddot{f}_{2}, \ddot{f}_{0}=0 \text { and }-4 f_{0}-\frac{1}{v_{s}^{2}} \ddot{f}_{1}=0 \\
\Rightarrow G=-\frac{8}{3} v_{s}^{2} f_{01} t^{3}-8 v_{s}^{2} f_{00} t^{2}+G_{1} t+G_{0}, \\
f_{0}=f_{01} t+f_{00} \\
\text { and } f_{1}=-\frac{2}{3} v_{s}^{2} f_{01} t^{3}-2 v_{s}^{2} f_{00} t^{2}+f_{11} t+f_{10},
\end{gathered}
$$

where $f_{01}, f_{00}, f_{11}, G_{1}$ and $G_{0}$ are integration constants. As $G=\frac{d}{d t} \ln \frac{A_{0}}{A_{00}} \Leftrightarrow A_{0}(t)=A_{00} \exp \left[\int G d t\right]$,

where $A_{00}$ is an integration constant, we can conclude that the flux function grows with

$A_{0}(t)=A_{00} \exp \left[-\frac{2}{3} v_{s}^{2} f_{01} t^{4}-v_{s}^{2} \frac{8}{3} f_{00} t^{3}+\frac{G_{1}}{2} t^{2}+G_{0} t\right]$

and from Eq. (17) we can present the leading order of the time dependence of the density $\rho$

$\rho \propto \exp \left[-\frac{2}{15} v_{s}^{2} f_{01} t^{5}\right]$.

We can conclude that the magnetic field will decay and the complete region around the null point will be evacuated of matter, or, in the case of a negative value of e.g. $f_{01}$, a catastrophic pile-up of matter and field strength will take place.

\subsection{Case II: Polytropic approach}

With $p=K \rho^{\gamma}, \gamma=\left(C-C_{p}\right) /\left(C-C_{V}\right)$, with $C_{p}$ and $C_{V}$ as heat capacities at constant pressure, respectively constant volume, and $C$ as the actual heat capacity (see Chandrasekhar (1957) or Emden (1907) for details of the polytropic change), we get the differential equation

$\ddot{\varphi}+(\gamma-1) \dot{\varphi} \Delta \varphi=0$.

If we insert the general solution $\varphi=f(\xi, t)-\frac{G}{2} x^{2}$ into the nonlinear mass continuity equation, Eq. (30) we get restrictions for the homogenous part $f(\xi, t)$ of the general solution and the inhomogenuous part of the solution represented by $G(t)$. The solution method is analogous to the isothermal case, so that we present here only briefly our preliminary results:

1. $\dot{G}=0$ and $f^{\prime \prime}=0$, exponentially unstable (interesting for flares); $G_{0}<0$ and $\gamma<1$ or $G_{0}>0$ and $\gamma>1$ delivers with respect to time unbounded solutions of the flow.
2. $\dot{G}=0, \dot{f}=0$ and $f^{\prime \prime} \neq 0, \varphi=f(\xi)-G_{0} x^{2} / 2$ (stationary flow); the magnetic field decays or grows exponentially.

3. $\dot{G} \neq 0$ and $f^{\prime \prime}=0$, (i) oscillating flux function, but diverging non-parallel parts of the flow (finite time singularity $\propto \tan (t)$ ) or (ii) unbounded parallel flow $\propto \cosh (t)$, but bounded flux function $\propto \tanh (t)$, or (iii) flux function and non-parallel flow velocity obey finite time singularity $\propto 1 /\left(t-t_{0}\right)$.

2.5 Illustrative example: Standard stagnation point of a vortex free velocity field

Again we want to use velocity fields that allow only for velocities that are small compared to the speed of sound on the linear scale around the stagnation point. We focus our attention now on the hydrodynamical aspect, i.e. the stagnation point. The velocity potential must have a form that guarantees the existence of a null point of the flow with a separatrix. For this the most general Ansatz is

$\varphi=a(t) x^{2}-b(t) y^{2}+c(t) x y+d(t) x+e(t) y$,

and inserting this into the nonlinear mass continuity equation, Eq. (30), we get at the end that $\dot{a} \propto \dot{b}$ and therefore $a(t) \propto t$ and $b(t) \propto t$. Thus the flow is unbounded with respect to the subsonic constraint and the needed fluid topology or is stationary with $a, b=$ const. The assumption $a=b$ leads to $\ddot{c}=0$ (the ordinary differential equation ist the same for the other coefficients) and therefore to an unbounded growth of the velocity field in the non-trivial case.

\subsection{Preliminary results for the 2-D case}

In the vicinity of magnetic null points several kinds of breakdown of the slow or quasi-static ideal MHD approach can occur: Either due to exponential instabilities/decay or other unbounded solutions or due to finite time singularities. These instabilities are useful for explaining the onset of eruptive processes on the solar surface. On the other hand no nontrivial and reasonable asymptotic stationary case can be found that can be used as a model of the vicinity of the heliospheric nose.

Many problems remain: is the appearance of these finite time singularities an inherent property of the dynamic in the vicinity of a null point? We would like to find solutions for the fully non-linear problem and use more general barotropic laws instead of a polytropic law in the future.

\section{Problems of 3-D null}

We now turn to the investigation of stationary 3-D null points. Assume that a function $X$ and a velocity field $\mathbf{w}$ exist, so that $\boldsymbol{E}+\mathbf{w} \times \boldsymbol{B}=\nabla X$. The existence of such fields 
$X$, w guarantees that magnetic topology is conserved, respectively the magnetic field is frozen with respect to $\mathbf{w}$ (Hornig and Schindler, 1996). To get stationary background states of the MHD fields in the vicinity of null points, respectively stagnation points, we have to solve the following equation $\boldsymbol{\nabla} \times \boldsymbol{E}=\mathbf{0} \equiv \boldsymbol{\nabla} \times(-\mathbf{w} \times \boldsymbol{B})$, i.e.

$-(\mathbf{w} \cdot \nabla) \boldsymbol{B}-(\boldsymbol{\nabla} \cdot \mathbf{w}) \boldsymbol{B}+(\boldsymbol{B} \cdot \nabla) \mathbf{w}=\mathbf{0}$.

This is the induction equation of ideal MHD, and in the following we analyse the structure of the solutions in the vicinity of singular points of flow and field.

\subsection{Heliopause $=$ hydropause}

First we analyse the case if the stagnation point and the corresponding fluid separatrix is regarded as heliopause. We calculate the magnetic field with the help of the ideal Ohm's law at the position of the stagnation point, i.e.

$\mathbf{0}=\nabla \times(\mathbf{w} \times \boldsymbol{B})_{\mathbf{w}=\mathbf{0}}=\stackrel{\leftrightarrow}{J}_{\mathbf{w}} \boldsymbol{B}$,

with $\overleftrightarrow{J}_{\mathbf{w}}:=-(\nabla \cdot \mathbf{w}) \stackrel{\leftrightarrow}{1}+(\nabla \mathbf{w})^{T}$. But there is the problem of the ambiguous meaning of $\mathbf{w}$ (plasma, electron, particle velocity, neutral particles). Thus the question arises if we can identify the magnetic flux transporting velocity field w with some plasma species velocity, i.e. what has to 'stagnate' = stop at the stagnation point? A better way to define such a boundary surface in a unique manner is maybe to identify the separatrix of the magnetic field, the magnetopause, with the heliopause.

\subsection{Heliopause $=$ magnetopause of the solar wind}

For that case in which we regard the correponding magnetic separatrix (= the magnetopause), defined and determined by the magnetic null point, as the heliopause, we get:

$\mathbf{0}=\nabla \times(\mathbf{w} \times \boldsymbol{B})_{\boldsymbol{B}=\mathbf{0}}=-(\mathbf{w} \cdot \nabla) \boldsymbol{B}=\stackrel{\leftrightarrow}{J}_{\boldsymbol{B}} \mathbf{w}$,

where $\stackrel{\leftrightarrow}{J}_{\boldsymbol{B}}:=(\nabla \boldsymbol{B})^{T}$ is the transposed Jacobian matrix of $\boldsymbol{B}$.

\subsection{Discussion}

If the rank $\left(\stackrel{J}{J}_{\mathbf{w}}\right)<3$, then either 2-D magnetic singularities occur, or the solution must have parallel poloidal components of magnetic and velocity field, see Nickeler and Karlický (2006). This statement also holds if the rank of $\overleftrightarrow{J}_{\boldsymbol{B}}$ is smaller than 3 . Either the velocity field in this case would diverge at the magnetic null point or the poloidal flow would be field aligned. If the rank $=3$, then 3-D allows only for magnetic field and velocity fields with a 'spiral null'('spiralfield-crossing'), see Titov and Hornig (2000), the so called SFC-flows. The matrix has complex Eigenvalues with nonvanishing real parts, a hint that these equilibrium flows are probably generally unstable in linear perturbation analysis. The 3-D magnetic null point in ideal MHD implies also that it is a 3-D stagnation point, therefore the magnetopause and the heliopause are at least locally identical: this result must be clearly seen in simulations if they approach quasi-stationary states. The matrix $\stackrel{\leftrightarrow}{J}_{\mathbf{w}}$ of the 3-D flow null point, the stagnation point, must also show a rank larger than 2 , otherwise the problem leads to the conclusion that the magnetic field is not unique at the stagnation point, respectively that the flow is completely field aligned. Thus we are able to infer that locally heliopause and magnetopause are identical and that in 3-D ideal MHD stagnation points also have to be null points of the magnetic field and vice versa.

\section{Conclusions}

In the first part we study all possible time evolutions in the vicinity of a 2-D magnetic null or stagnation point. We calculate for the isothermal and polytropic case the complete solution space of the system of the ideal (M)HD equations. No bounded stationary solutions in the vicinity of such stagnation points or magnetic null points can be found, only purely field aligned flows, see Nickeler and Karlický (2006). This implies that due to the symmetry $\partial / \partial z=0$, the conserved quantity, the constant electric field in $z$-direction, leads to singular configurations. For the non-stationary case it is obvious that with respect to time no bounded or non-decaying solutions with small velocity exist at 2-D stagnation points (magnetic null points) within the framework of ideal (M)HD. Thus the solutions are unstable or represent collapse processes.

In the second part we extend our investigations to 3-D. We find that for the 3-D stationary case the magnetic null point must also be a null point of the flow, i.e. a stagnation point. Based on the results of Titov and Hornig (2000) we can conclude, that the 'fan' plane, i.e. the 2-D manifold (magnetic separatrix $=$ magnetopause) of the magnetic null point is identical to the fluid separatrix (=classical heliopause). This implies that the tangential surface of the magnetopause (between the solar wind magnetic field and the interstellar magnetic field) and the heliopause are identical at the stagnation point. The magnetopause around the stagnation point(= magnetic null point) is therefore at least locally identical to the heliopause. The situation in 3-D allows only for a special class of stationary solutions: Either the flow must be completely field-aligned or must be a so called SFCflow, found by Titov and Hornig (2000). In the future it has to be checked whether these flows are stable to small perturbations to see, whether ideal MHD can be used to model quasi-stationary states for the heliosphere. It has therefore also to be checked whether such states can be found in simulations or whether unphysical reconnection regimes can be found, although ideal MHD is used. If the latter is the case then one has to find and use an Ohm's law, which allows for reconnection at the heliospheric nose. 
Acknowledgements. We acknowledge financial support by the Grant IAA300030701 and IAA300030804 of Grant Agency of the Academy of Sciences of the Czech Republic.

Edited by: V. V. Izmodenov

Reviewed by: M. Ruderman and another anonymous referee

\section{References}

Arnold, V. I.: Ordinary differential equations, Springer, Berlin, 1992.

Baranov, V. B., Fahr, H.-J., and Ruderman, M. S.: Investigation of macroscopic instabilities at the heliopause boundary surface, Astron. Astrophys., 261, 341-347, 1992.

Belov, N. A.: Instability of a tangential discontinuity in a twodimensional flow with a stagnation point, Fluid Dynamics, 32(2), 219-222, 1997.

Bronstein, I. N. and Semendjajew, K. A.: Taschenbuch der Mathematik, Verlag Harri Deutsch, Thun und Frankfurt/Main, 1979.

Chandrasekhar, S.: An introduction to the study of stellar structure, Dover Publications, New York, 1957.

Contopoulos, J.: General Axissymmetric Magnetohydrodynamic Flows: Theory and Solutions, Astrophys. J., 460, 185-198, 1996.

Emden, R.: Gas balls: Applications of the mechanical heat theory to cosmological and meteorological problems, Teubner, 1907.

Fahr, H.-J., Fichtner, H., and Scherer, K.: Determination of the heliospheric shock and of the supersonic solar wind geometry by means of the interstellar wind parameters, Astron. Astrophys., 277, 294-264, 1993.

Fahr, H.-J., Grzedzielski, S., and Ratkiewicz, R.: Magnetohydrodynamic modeling of the 3-dimensional heliopause using the Newtonian approximation, Ann. Geophysicae, 6, 337-354, 1988.

Hornig, G. and Schindler, K.: The problem of magnetic topology and its invariant definition, Phys. of Plasmas, 3(3), 781-791, 1996.

Nickeler, D. H., Goedbloed, J. P., and Fahr, H.-J.: Stationary fieldaligned MHD flows at astropauses and in astrotails, Astron. Astrophys., 454, 797-810, 2006.

Nickeler, D. and Karlický, M.: Are heliospheric flows magnetic flux- or line conserving?, Astrophys. Space Sci. Trans., 2, 6372, 2006

Parker, E. N.: Interplanetary dynamical processes, Interscience Publishers, New York, 1963.

Parnell, C. E., Smith, J. M., Neukirch, T., and Priest, E. R.: The structure of three-dimensional magnetic neutral points, Phys. of Plasmas, 3(3), 759-770, 1996.

Reitmann, V.: Reguläre und chaotische Dynamik, Teubner, Stuttgart Leipzig, 1996.

Ruderman, M. S.: Absolute and convective instability of tangential discontinuities in viscous fluids: Application to heliopause, Astrophys. and Space Science, 274, 327-341, 2000.

Titov, V. S. and Hornig, G.: Magnetohydrodynamic flows sustaining stationary magnetic nulls, Phys. of Plasmas, 7, 3542-3550, 2000.

Zank, G.: private communication, 2007. 